\title{
THE CONORMAL TORUS IS A COMPLETE KNOT INVARIANT
}

\author{
VIVEK SHENDE ${ }^{\circledR}$ \\ Departments of Mathematics, U.C. Berkeley, Berkeley CA 94720, USA; \\ email: vivek@math.berkeley.edu
}

Received 7 September 2017; accepted 18 May 2019

\begin{abstract}
We use microlocal sheaf theory to show that knots can only have Legendrian isotopic conormal tori if they themselves are isotopic or mirror images.
\end{abstract}

2010 Mathematics Subject Classification: 57M25, 57M27, 55N30

\section{Introduction}

A knot $K \subset \mathbb{R}^{3}$ determines, by taking the unit conormals, a torus in the unit cosphere bundle. By general position arguments, the smooth type of this embedding $\mathbb{T}_{K} \subset S^{*} \mathbb{R}^{3}$ knows nothing about the knot. However, this embedding is that of a Legendrian in a contact manifold, and a smooth isotopy of knots $K \sim K^{\prime}$ induces a Legendrian isotopy of conormals $\mathbb{T}_{K} \sim \mathbb{T}_{K^{\prime}}$. Thus, the Legendrian isotopy type of $\mathbb{T}_{K}$ is a topological invariant of $K$.

More generally, taking the cosphere or cotangent bundle gives a natural functor from smooth topology to contact or symplectic geometry, and it is a fundamental question in these subjects to understand what this functor remembers and what it forgets. A representative example is Arnold's conjecture that every compact exact Lagrangian in the cotangent bundle is Hamiltonian isotopic to the zero section; there has been much recent work in this direction [N1, FSS, Abo, AK].

The question regarding the extent to which $\mathbb{T}_{K}$ determines $K$ has previously been studied by holomorphic curve techniques, using the relative contact

(C) The Author 2019. This is an Open Access article, distributed under the terms of the Creative Commons Attribution-NonCommercialShareAlike licence (http://creativecommons.org/licenses/by-nc-sa/4.0/), which permits noncommercial re-use, distribution, and reproduction in any medium, provided the same Creative Commons licence is included and the original work is properly cited. The written permission of Cambridge University Press must be obtained for commercial re-use. 
homology of the pair $\left(S^{*} \mathbb{R}^{3}, \mathbb{T}_{K}\right)$. Perhaps, the first indication of the true strength of this invariant was in [Ng3], where it was shown to detect the unknot. More recently, this was extended to torus knots [GLi].

In the present article, we take a different approach to the study of $\mathbb{T}_{K}$. In general, one can study the geometry of Legendrians in cosphere bundles by assigning, to a Legendrian $\Lambda \subset S^{*} M$, the category of sheaves with microsupport in $\Lambda$. This approach reaches back in some sense to the microlocal analysis of Sato and Hörmander, is technically built on the microlocal sheaf theory of Kashiwara and Schapira [KS], and was applied to study symplectic geometry by Tamarkin [Tam] and subsequent authors [GKS, Gui, Gui2, Gui3, Chi, STZ, STWZ, STW]. Using this method, we give here the first proofs of the following results.

THEOREM 1. Let $L, L^{\prime}$ be oriented nonsplit links in $\mathbb{R}^{3}$. If there is a parameterized Legendrian isotopy $\mathbb{T}_{L} \rightarrow \mathbb{T}_{L^{\prime}}$, then there is a topological isotopy $L \rightarrow L^{\prime}$.

THEOREM 2. For knots $K, K^{\prime}$ in $\mathbb{R}^{3}$, if there is any Legendrian isotopy $\mathbb{T}_{K} \rightarrow$ $\mathbb{T}_{K^{\prime}}$, then the knots are either isotopic or mirror.

In the first theorem, by 'parameterized isotopy', we mean that the isotopy respects the homotopy classes of the meridians and longitudes of the tori; we do not impose this condition in the second theorem. Also, in the first theorem, by nonsplit, we mean that no sphere separates some components of the link from some others.

The strategy of proof is as follows. A standard consequence of Gray's stability theorem [Gr] is that a Legendrian isotopy is induced by an ambient contact isotopy, which can moreover be chosen to be constant away from an open set around the Legendrian isotopy. Henceforth, by Legendrian isotopy, we will always implicitly mean such an extension. The sheaf quantization of this contact isotopy [GKS] gives an equivalence of derived categories $s h_{\mathbb{T}_{K}}\left(\mathbb{R}^{3}\right) \cong$ $s h_{\mathbb{T}_{K^{\prime}}}\left(\mathbb{R}^{3}\right)$.

These categories of sheaves carry information about the fundamental group of the knot complement, which (when marked by the peripheral subgroup) determines the knot [Wal]. Nevertheless, extracting this information is a nontrivial problem: the derived category of local systems on the complement is only a piece of $s h_{\mathbb{T}_{K}}\left(\mathbb{R}^{3}\right)$. Moreover, it is not generally possible to determine a ring from its abelian category of modules, and it is not generally possible to extract an abelian category from its derived category. For instance, the existence and significance of derived equivalences in birational algebraic geometry 
[Orl, BO] is a subtle and fascinating subject. In fact, we do not know whether a derived equivalence of knots $s h_{\mathbb{T}_{K}}\left(\mathbb{R}^{3}\right) \cong s h_{\mathbb{T}_{K^{\prime}}}\left(\mathbb{R}^{3}\right)$ forces $K$ to be isotopic to $K^{\prime}$.

Instead, we use additional geometric information to rigidify this isomorphism. The key observation is that because we are working in the noncompact $\mathbb{R}^{3}$, the isotopy can be pushed off the fiber at a point. This point provides a fiber functor which rigidifies our categories sufficiently to eventually extract the group ring. In particular, we do not know whether the result still holds with $\mathbb{R}^{3}$ replaced by $S^{3}$. This would follow if it was shown that no nontrivial derived equivalences of knots could exist.

To finish the proof, we appeal to known results in 3-manifold topology. First, the fundamental group of a link complement is left-orderable [HS, Lemma 2] and, hence, can be recovered from its integral group ring. Finally, by the work of Waldhausen [Wal], the fundamental group of the complement together with the classes of the meridians and longitudes determines the link.

REMARK 1. An approach to these results using Legendrian contact homology now exists [ENS]. It relies on [CELN], which amounts to a comparison theorem between sheaves and $\mathrm{LCH}$ in this setting. Other comparisons can be extracted from [Cor1, Cor2], and [BEY].

\section{Some review of sheaf theory}

We first recall some basic facts about sheaves; for details, see, for example, [KS, Chs. 1,2]. For a topological space $T$, a presheaf is a functor from the category whose objects are open sets and whose morphisms are inclusions. A sheaf is such a functor satisfying certain locality conditions. We write $\operatorname{Sh}(T)$ for the category of sheaves of $\mathbb{Z}$-modules on $T$.

The category $\operatorname{Sh}(T)$ is abelian, and one may form its derived category $\operatorname{sh}(T)$. By definition, this is the localization of the category of complexes of sheaves along the quasi-isomorphisms. In our main foundational reference [KS], the authors work with the bounded derived category and regard it as a triangulated category. It is more typical these days to work in the unbounded derived category and, moreover, to regard derived categories as dg categories. All sheaves which will appear here are in fact bounded, but it is never important for us that they are bounded (except that strictly speaking [KS] requires this). All arguments we make in fact only require the triangulated structure, but at some points, it is more natural to have a dg point of view, for example, to formulate the statement (which we will not use) that derived endomorphisms of the stalk functor identify $\operatorname{loc}(M) \cong C_{*}(\Omega M)-$ mod. Henceforth, by 'sheaf', we mean an object of 
the derived category sh(T) unless explicitly specified otherwise. All functors between sheaf categories will mean the derived functors; the word isomorphism in this context means quasi-isomorphism. We also adopt the convention that we write $=$ to mean an isomorphism so canonical that no confusion can possibly arise.

Given a map $f: T \rightarrow S$, there is an adjoint pair of (derived) pullback and pushforward functors $f^{*}: \operatorname{sh}(S) \leftrightarrow s h(T): f_{*}$. For locally compact spaces, there are also 'compact support' versions $f_{!}: \operatorname{sh}(T) \leftrightarrow \operatorname{sh}(S): f^{!}$.

For a sheaf $\mathcal{F}$ on $S$, we will often abbreviate $f^{*} \mathcal{F}$ as $\left.\mathcal{F}\right|_{T}$, especially when $T$ is a subset of $S$. In particular, for a point $s \in S$, we write $\left.\mathcal{F}\right|_{s}$ for the stalk at $s$.

For an open subset $u: U \subset S$, the open subsets of $U$ are amongst the open subsets of $S$, and the maps $u^{*}, u^{!}: \operatorname{sh}(S) \rightarrow \operatorname{sh}(U)$ agree and are given by $\left(u^{*} \mathcal{F}\right)(O)=\mathcal{F}(O)$. In particular, for $s \in U$, there is a canonical isomorphism

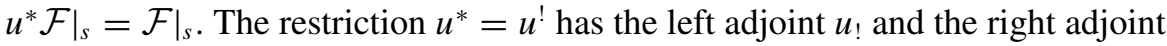
$u_{*}$, both of which are fully faithful. The functor $u_{!}$is the 'extension by zero': one has

$$
\left.u_{!} \mathcal{G}\right|_{s}= \begin{cases}\left.\mathcal{G}\right|_{s} & \text { if } s \in U, \\ 0 & \text { otherwise }\end{cases}
$$

In fact, the same holds for the inclusion of any locally closed subset.

For a closed subset $v: V \subset S$, the functors $v_{!}, v_{*}: \operatorname{sh}(V) \rightarrow \operatorname{sh}(S)$ agree and thus, in particular on stalks, behave as dictated by the above formula. These functors are fully faithful.

Consider a decomposition of $S$ into an open set $U$ and its closed complement $V$. For any $\mathcal{F} \in \operatorname{sh}(S)$, there is an exact triangle

$$
u ! u^{!} \mathcal{F} \rightarrow \mathcal{F} \rightarrow v_{*} v^{*} \mathcal{F} \stackrel{[1]}{\rightarrow}
$$

The first two maps arise from the adjunctions $\left(u_{!}, u^{!}\right)$and $\left(v^{*}, v_{*}\right)$. The fact that the sequence determines an exact triangle is easily checked on stalks after rewriting the sequence as

$$
u_{!} u^{*} \mathcal{F} \rightarrow \mathcal{F} \rightarrow v_{!} v^{*} \mathcal{F} \stackrel{[1]}{\rightarrow}
$$

Indeed, it is clear from Equation (1) that for stalks over $U$, the first map is an isomorphism and the second is zero, and for stalks over $V$, the reverse is true. This sequence is a sheaf-theoretic version of the long exact sequence in cohomology.

Note additionally that

$$
\operatorname{Hom}_{s h(S)}\left(u ! \mathcal{G}, v_{*} \mathcal{H}\right)=\operatorname{Hom}_{s h(U)}\left(v^{*} u ! \mathcal{G}, \mathcal{H}\right)=\operatorname{Hom}_{s h(U)}(0, \mathcal{H})=0
$$


for any $\mathcal{G} \in \operatorname{sh}(U)$ and $\mathcal{H} \in \operatorname{sh}(V)$. In short, identifying $\operatorname{sh}(U)$ with its image under $u_{!}$and, likewise, $\operatorname{sh}(V)$ with its image under $v_{*}=v_{!}$, there is a semiorthogonal decomposition

$$
\operatorname{sh}(S)=\langle\operatorname{sh}(V), \operatorname{sh}(U)\rangle .
$$

This decomposition has been exploited to a great effect in algebraic geometry and geometric representation theory since [BBD].

By definition, a sheaf on $S$ is constant if it is isomorphic to the pullback of some sheaf on a point. A sheaf is locally constant if this holds on some cover of $S$. We write $\operatorname{loc}(S)$ for the category of locally constant sheaves on $S$ or, in other words, what would classically have been termed complexes of sheaves with locally constant cohomology.

We turn now to the notion of microsupport [KS, Ch. 5]. To a sheaf $\mathcal{F}$ on $M$, there is a closed conical locus $s s(\mathcal{F}) \subset T^{*} M$-the directions along which the sections of the sheaf fail to propagate. To a closed subset $\Lambda \subset T^{*} M$, we associate the category $s h_{\Lambda}(M)$ whose objects have microsupport contained in $\Lambda$. It is, by definition, a full subcategory of the (derived) category of sheaves on $M$.

We write $T^{\circ} M=T^{*} M \backslash M$ for the complement of the zero section. When we consider conical loci containing the entire zero section, we often specify them by naming only their intersection $T^{\circ} M$ or, equivalently, their images in $T^{\circ} M / \mathbb{R}_{>0}=S^{*} M$. By abuse of notation, for a closed subset $\Lambda \subset S^{*} M$, we write $s h_{\Lambda}(M):=s h_{\text {Cone }(\Lambda) \cup T_{M}^{*} M}(M)$.

A sheaf $\mathcal{K}$ on $M \times M^{\prime}$ can be used as an 'integral kernel' to define a functor

$$
\begin{aligned}
\mathcal{K} \star: \operatorname{sh}(M) & \rightarrow \operatorname{sh}\left(M^{\prime}\right) \\
\mathcal{F} & \mapsto \pi_{M^{\prime} !}\left(\mathcal{K} \otimes \pi_{M}^{*} \mathcal{F}\right) .
\end{aligned}
$$

Here, $\pi_{M}$ and $\pi_{M^{\prime}}$ are the projections to the factors of $M \times M^{\prime}$. We denote analogously by $\pi_{T^{*} M}$ and $\pi_{T^{*} M^{\prime}}$ the projections of $T^{*}\left(M \times M^{\prime}\right)=T^{*} M \times T^{*} M^{\prime}$ to its factors.

Assuming $\operatorname{ss}(\mathcal{K}) \rightarrow T^{*} M^{\prime}$ is proper, the microsupport of the integral transform is contained in the set-theoretic integral transform of the microsupports [KS, Proposition 7.1.2]:

$$
s s(\mathcal{K} \star \mathcal{F}) \subset \pi_{T^{*} M^{\prime}}\left(s s(\mathcal{K}) \cap \pi_{T^{*} M}^{-1}(s s(\mathcal{F}))\right) .
$$

Of particular interest is the case when $\operatorname{ss}(\mathcal{K}) \cap T^{\circ} M \times T^{\circ} M$ is a graph of a (conic) symplectomorphism $T^{\circ} M \rightarrow T^{\circ} M$; equivalently, the symplectization of a contactomorphism $S^{*} M \rightarrow S^{*} M$. Kernels with such microsupport induce equivalences [KS, Theorem 7.2.1].

In fact, there is a canonical way to associate such a kernel to any contact isotopy. 
THEOREM 3 [GKS]. Let $\phi_{t}: S^{*} M \rightarrow S^{*} M$ be a compactly supported contact isotopy, for $t \in I$. Then there is a unique kernel $\Phi \in \operatorname{sh}(M \times M \times I)$ such that $\left.\operatorname{ss}\left(\Phi_{t}\right)\right|_{T^{\circ} M \times T^{\circ} M}$ is the graph of $\phi_{t}$ and $\Phi_{0}$ is the constant sheaf on the diagonal. The resulting integral transformation $\Phi_{t} \star$ is an equivalence of categories for all $t$.

Moreover, if $m \in M \backslash \operatorname{Supp}\left(\phi_{t}\right)$, then in some neighborhood $U_{m} \ni m$, the sheaf $\left.\Phi\right|_{U_{m} \times M \times I}$ is the constant sheaf on the diagonal.

Let us sketch a proof. Any contact isotopy is induced by a (time-varying) contact Hamiltonian. It is easy to see that one can use a composite of an isotopy induced by a positive Hamiltonian with the one induced by a negative Hamiltonian, so it suffices to prove the result for positive contact isotopies. By composition, it suffices to prove the result for arbitrarily small positive isotopies. The graph of such an isotopy is the inward conormal to a small neighborhood of the diagonal in $M \times M$. The only sheaf with this conormal which is isotopic to the diagonal (through sheaves with such conormals) is the star pushforward of the constant sheaf on the interior of this neighborhood.

Recall that if $(M, g)$ is a Riemannian manifold, then the Reeb flow associated with the natural Liouville contact form on the cosphere bundle is identified with the geodesic flow by the metric. For this particular flow, the sheaf quantization is easily described.

COROllary 4. Let $(M, g)$ be a Riemannian manifold. Then for $T$ smaller than the injectivity radius of $g$, the integral kernel for the sheaf quantization of the Reeb flow for time $T$ is given by the constant sheaf on the locus

$$
\{(x, y)|| x-y \mid \leqslant T\} .
$$

Proof. Consider the constant sheaf on the locus $\{(x, y, t)|| x-y \mid \leqslant t\}$ in $M \times$ $M \times[0, T]$. At $t=0$, this is precisely the constant sheaf on the diagonal, and a direct computation shows that it has the correct microsupport. By the uniqueness in Theorem 3, its slices give the correct kernels.

COROLlary 5. In the setting of the previous corollary, let $\Phi_{T}$ be the sheaf quantization of the time $T$ Reeb flow. Let $N \subset M$ be a submanifold with no selfgeodesics of length smaller than $T$. Let $\operatorname{Nbd}_{T}(N)$ denote the (closed) locus of points at distance at most $T$ from $N$. Then

$$
\Phi_{T} \mathbb{Z}_{N}=\mathbb{Z}_{N b d_{T}(N)} \quad \Phi_{T} \mathbb{Z}_{M \backslash N}=\mathbb{Z}_{M \backslash N b d_{T}(N)} .
$$




\section{Recovering the fundamental group}

We apply Theorem 3 as follows. For a submanifold $Y \subset X$, let $\mathbb{T}_{Y} \subset S^{*} X$ denote the unit conormal bundle.

Corollary 6. Let $N, N^{\prime}$ be compact submanifolds of $M$. A Legendrian isotopy of conormal tori $\mathbb{T}_{N} \rightsquigarrow \mathbb{T}_{N^{\prime}}$ induces an equivalence of categories $s h_{\mathbb{T}_{N}}(M) \cong s h_{\mathbb{T}_{N^{\prime}}}(M)$.

That is, the category $s h_{\mathbb{T}_{N}}(M)$ depends only on the Legendrian isotopy type of $\mathbb{T}_{N}$.

In Theorem 3, it is essential to work with the derived category of sheaves (or a dg enhancement), rather than the abelian category, where the theorem would simply be false. However, derived categories tend to have many autoequivalences, making them slippery invariants. When $M$ is noncompact, the situation is more rigid.

THEOREM 7. Let $N, N^{\prime}$ be compact submanifolds of a noncompact manifold $M$. The sheaf quantization $s h_{\mathbb{T}_{N}}(M) \cong s h_{\mathbb{T}_{N^{\prime}}}(M)$ of a Legendrian isotopy of conormals satisfies the following:

- $\operatorname{loc}(N)$ is carried to $\operatorname{loc}\left(N^{\prime}\right)$;

- $\operatorname{loc}(M \backslash N)$ is carried to $\operatorname{loc}\left(M \backslash N^{\prime}\right)$;

- the latter isomorphism preserves abelian categories of local systems.

Moreover, there is an isomorphism of integral group rings $\mathbb{Z}\left[\pi_{1}(M \backslash N)\right] \cong$ $\mathbb{Z}\left[\pi_{1}\left(M \backslash N^{\prime}\right)\right]$.

Proof. We begin with the isomorphism from the corollary, $s h_{\mathbb{T}_{N}}(M) \cong$ $s h_{\mathbb{T}_{N^{\prime}}}(M)$. Note that by [KS, Proposition 8.4.1], the category $s h_{\mathbb{T}_{N}}(M)$ is just the category of sheaves constructible with respect to the stratification

$$
M=(M \backslash N) \cup N .
$$

That is, $\mathcal{F} \in s h_{\mathbb{T}_{N}}(M)$ if and only if $\left.\mathcal{F}\right|_{M \backslash N}$ and $\left.\mathcal{F}\right|_{N}$ are (cohomologically) locally constant.

Let $i: N \hookrightarrow M \hookleftarrow M \backslash N: j$ be the closed and open inclusions. As we recalled in the previous section, the functors $i_{!}=i_{*}$ and $j$ ! give a semiorthogonal decomposition $\operatorname{sh}(M)=\langle\operatorname{sh}(M \backslash N), \operatorname{sh}(N))\rangle$. Imposing the microsupport condition, we have

$$
s h_{\mathbb{T}_{N}}(M)=\langle\operatorname{loc}(N), \operatorname{loc}(M \backslash N)\rangle .
$$


In particular, $\operatorname{loc}(M \backslash N)$ is characterized as the left orthogonal complement to $\operatorname{loc}(N)$ in $s h_{\mathbb{T}_{N}}(M)$, that is, the objects with no homs to any object of $\operatorname{loc}(N)$. Likewise, $\operatorname{loc}(N)$ is characterized as being the right orthogonal complement to $\operatorname{loc}(M \backslash N)$.

Fix a compactly supported contact isotopy inducing the Legendrian isotopy and choose $m \in M$ of the complement of the projection of this compact set (in particular, in the complement of $N, N^{\prime}$ ). Denote by $\Phi$ the [GKS] equivalence induced by the isotopy. As $m$ is outside the support of $\phi$, the kernel of $\Phi$ is the constant sheaf on the diagonal above a neighborhood of $m$; hence, $\Phi$ commutes with the (derived) functor of taking stalk at $m$.

As per the discussion around the semiorthogonal decomposition, the full subcategory $\operatorname{loc}(N)$ of $s h_{\mathbb{T}_{N}}(M)$ is characterized as the kernel of the restriction $j^{*}=j^{!}$. As sheaves in $s h_{\mathbb{T}_{N}}(M)$ are locally constant in $M \backslash N$, this restriction is zero iff the stalk at $m \in M \backslash N$ is zero. That is, $\operatorname{loc}(N)$ is characterized as the full subcategory of $s h_{\mathbb{T}_{N}}(M)$ with acyclic stalk at $m$. The same holds for $\operatorname{loc}\left(N^{\prime}\right) \subset s h_{\mathbb{T}_{N}^{\prime}}(M)$.

Since $\Phi$ commutes with taking the stalk at $m$, it preserves this property and, hence, carries $\operatorname{loc}(N)$ to $\operatorname{loc}\left(N^{\prime}\right)$. Hence, $\Phi$ carries also the left orthogonal complement of one of these to the left orthogonal complement of the other, that is, carries $\operatorname{loc}(M \backslash N)$ to $\operatorname{loc}\left(M \backslash N^{\prime}\right)$. Noting again that $\Phi$ commutes with taking stalk at $m$, we see that $\Phi$ carries the subcategory of $\operatorname{loc}(M \backslash N)$ with stalk cohomology concentrated in degree zero to the corresponding subcategory of $\operatorname{loc}\left(M \backslash N^{\prime}\right)$.

Any (complex of) sheaf whose stalks have cohomology concentrated in degree zero is quasi-isomorphic to its zeroeth cohomology sheaf (here, sheaf can be taken in the nonderived sense). Consequently, the subcategory of $\operatorname{loc}(M \backslash N)$ whose stalks have cohomology in degree zero is the category of local systems on $M \backslash N$ in the nonderived sense, that is, the abelian category of modules over $\mathbb{Z}\left[\pi_{1}(M \backslash N)\right]$. (If one is thinking of $s h$ as a dg rather than merely triangulated category, one should take the homotopy category of this subcategory.)

Note that the identification of local systems with modules depends on the choice of a point, here $m$, and taking the stalk at $m$ corresponds the forgetful functor $\mathbb{Z}\left[\pi_{1}(M \backslash N)\right]$-mod $\rightarrow \mathbb{Z}$-mod.

Thus, we have an equivalence $\mathbb{Z}\left[\pi_{1}(M \backslash N)\right]$-mod $\cong \mathbb{Z}\left[\pi_{1}\left(M \backslash N^{\prime}\right)\right]$-mod, commuting with the forgetful functor to $\mathbb{Z}$-mod. But any ring $A$ can be recovered as the endomorphisms of the forgetful functor $A$-mod $\rightarrow \mathbb{Z}$-mod: this functor is co-represented by the left $A$-module $A$, whose endomorphisms are $A$ acting by right multiplication.

REMARK 2. Note that we have not assumed even $\operatorname{dim} N=\operatorname{dim} N^{\prime}$. 
REMARK 3. This argument requires the use of infinite $\mathbb{Z}$-rank local systems, specifically the local system with fiber $\mathbb{Z}\left[\pi_{1}(M \backslash N)\right]$.

REMARK 4. By not passing to the abelian category, one would learn that, in fact, the (homotopy) rings of chains on the based loop space are isomorphic: $C_{*}(\Omega(M \backslash N), \mathbb{Z}) \cong C_{*}\left(\Omega\left(M \backslash N^{\prime}\right), \mathbb{Z}\right)$. One could still learn more by working over the sphere spectrum rather than $\mathbb{Z}$; it is known to experts that the microlocal sheaf theory works here, although the details have not appeared in the literature.

It is not generally true that $\mathbb{Z}[G]$ determines $G$; there are counterexamples even amongst finite groups [Her]. The most basic difficulty, studied by Higman in his thesis [Hig], is that $\mathbb{Z}[G]$ can have nontrivial units, that is, those which are not $\pm g$ for $g \in G$. He conjectured (and it remains open) that a torsion-free group has no nontrivial units; he also observed that imposing the much stronger condition of left-orderability ensures that there are no nontrivial units. Note that such a group can be recovered from $\mathbb{Z}[G]$ as the quotient of the units by the torsion units, since as $G$ is torsion-free and there are no nontrivial units, the only torsion units are \pm 1 .

REMARK 5. In light of the previous remark, it is natural to ask whether Hertling's counterexample still works for the sphere-spectrum group ring.

Fortunately, it is known that the fundamental group of a nonsplit link complement is locally indicable and, hence, left-orderable [HS]. We conclude the following.

THEOREM 8. If $L, L^{\prime} \subset \mathbb{R}^{3}$ are links with Legendrian isotopic conormal tori, then their complements have isomorphic fundamental groups.

REMARK 6. One might want to try and deduce an isomorphism of knot groups instead by showing that the equivalence of categories preserved the monoidal structure. However, this does not follow from the [GKS] construction: the isomorphism is constructed as a convolution, and convolution does not generally respect the tensor product. Moreover, along the isotopy, the Legendrian is not generally a conormal, and in this case, the category $s h_{\Lambda}\left(\mathbb{R}^{3}\right)$ is not closed under tensor product.

From this, it already follows that the conormal torus distinguishes amongst prime knots by the work of Waldhausen [Wal] and Gordon and Luecke [GL, Corollary 2.1]. 


\section{Recollections on $\mu \mathcal{H}$ om}

In what follows, we will use the microlocalization functor introduced in [KS, Section 4.4].

$$
\mu \mathcal{H} \text { om }: \operatorname{sh}(X)^{\mathrm{op}} \times \operatorname{sh}(X) \rightarrow \operatorname{sh}\left(T^{*} X\right) .
$$

It restricts to the usual $\mathcal{H o m}$ sheaf along the zero section: $\mu \mathcal{H}$ om $(\mathcal{F}$, $\mathcal{G})\left.\right|_{X}=\mathcal{H} \operatorname{om}(\mathcal{F}, \mathcal{G})[\mathrm{KS}$, Theorems 4.3.2.iv, 4.4.2.i]. It is supported along the intersection of microsupports of the original sheaves: $\operatorname{supp}(\mu \mathcal{H}$ om $(\mathcal{F}, \mathcal{G})) \subset$ $s s(\mathcal{F}) \cap s s(\mathcal{G})$ [KS, Corollary 5.4.10].

A crucial property of this functor is that microlocalization commutes with sheaf quantization. More precisely, from [KS, Equation 7.2.5], we see that given a contactomorphism $\phi: S^{*} X \rightarrow S^{*} X$ and its sheaf quantization $\Phi: \operatorname{sh}(X) \rightarrow$ $\operatorname{sh}(X)$,

$$
\left.\phi_{*} \mu \mathcal{H o m}(\mathcal{F}, \mathcal{G})\right|_{S^{*} X}=\left.\mu \mathcal{H} \operatorname{om}(\Phi \mathcal{F}, \Phi \mathcal{G})\right|_{S^{*} X} .
$$

We require only the following two elementary calculations of $\mu \mathcal{H}$ om:

LeMma 9. Let $X$ be a manifold.

(1) Let $i: Y \subset X$ be the inclusion of a closed submanifold without boundary and let $\mathcal{L}, \mathcal{M}$ be local systems on $Y$. Then $\operatorname{ss}(\mathcal{L})=T_{Y}^{*} X=\operatorname{ss}(\mathcal{M})$; and if $\pi: T_{Y}^{*} X \rightarrow X$ is the projection, $\mu \mathcal{H o m}\left(i_{*} \mathcal{L}, i_{*} \mathcal{M}\right)=\pi^{*} \mathcal{H o m}(\mathcal{L}, \mathcal{M})$.

(2) Let $j: U \rightarrow X$ be the inclusion of an open submanifold whose closure is a manifold with boundary, and let $\mathcal{L}, \mathcal{M}$ be local systems on $U$. Then $s s(\mathcal{L})=T_{U}^{+} X=s s(\mathcal{M})$, where + means we take the outward conormal vectors along the boundary. Moreover, if $\pi: T_{U}^{+} X \rightarrow X$ is the projection, $\mu \mathcal{H o m}\left(i_{*} \mathcal{L}, i_{*} \mathcal{M}\right)=\pi^{*} \mathcal{H o m}(\mathcal{L}, \mathcal{M})$.

Proof. The assertions regarding the microsupport are [KS, Propositions 5.3.2 and 5.3.3]. As the $\mu \mathcal{H}$ om is supported on the microsupport and its restriction to the zero section is the usual sheaf $\mathcal{H o m}$, it suffices to show that in these cases, the $\mu \mathcal{H o m}$ is locally constant on its support. This is a local calculation; hence, we may take $\mathcal{M}, \mathcal{L}$ to be the constant sheaf. The required calculation in the first case follows from [KS, Proposition 4.4.3], together with the observation that the Fourier transform of the constant sheaf on the zero section of some bundle is the constant sheaf supported everywhere on the dual bundle. The second case reduces to (a product of a trivial factor with) the sheaf $\mathbb{Z}_{(0, \infty)}$ on $\mathbb{R}$. This remaining one-dimensional calculation is a pleasant exercise. 


\section{Longitudes and meridians}

To establish our main result, we should show that the isomorphism established in Theorem 7 respects the 'peripheral subgroups', that is, the images of the fundamental groups of the boundary tori of $\mathbb{R}^{3} \backslash L$ and of $\mathbb{R}^{3} \backslash L^{\prime}$. Note that once we have done so, we need only appeal to [Wal] and not additionally to [GL]. Our main tool will be the microlocal hom functor recalled in the previous section.

For the most part, we work in the general setting of Theorem 7: $M$ is a noncompact manifold, $N, N^{\prime}$ are compact submanifolds, $\phi_{t}: S^{*} M \rightarrow S^{*} M$ is a contact isotopy with $\phi_{1}\left(\mathbb{T}_{N}\right)=\mathbb{T}_{N^{\prime}}$, and $m \in M$ is a point away from the projection of the isotopy. Let $\Phi_{t}: \operatorname{sh}(M) \rightarrow \operatorname{sh}(M)$ be the sheaf quantization of the isotopy.

LEMMA 10. $\Phi_{1}$ acts trivially on $\operatorname{loc}(M)$.

Proof. This has nothing to do with noncompactness of $M$. Fix some $\mathcal{F} \in \operatorname{loc}(M)$. We apply the kernel for the whole family $\Phi_{t}$ to obtain a sheaf on $M \times[0,1]$. By the compatibility of microsupports with contact transformation (Equation (3)), this sheaf is locally constant. Let $\pi: M \times[0,1] \rightarrow M$ be the projection. We claim that any locally constant sheaf $\mathcal{G}$ on $M \times[0,1]$ is isomorphic to the pullback of a locally constant sheaf on $M$. Indeed, there is a natural map $\pi^{*} \pi_{*} \mathcal{G} \rightarrow \mathcal{G}$, which is obviously an isomorphism locally on $M$, hence, an isomorphism. Thus, $\mathcal{F}=\Phi_{0} \mathcal{F}=\left.\left.\Phi_{t} \mathcal{F}\right|_{M \times 0} \cong \Phi_{t} \mathcal{F}\right|_{M \times 1} \cong \Phi_{1} \mathcal{F}$.

LEMMA 11. $\Phi_{1}\left(\mathbb{Z}_{N}\right) \cong \mathbb{Z}_{N^{\prime}}$.

Proof. We know that $\Phi_{1}\left(\mathbb{Z}_{N}\right)$ is (the pushforward of) a local system $\mathcal{L}$ on $N^{\prime}$. Using Equation (4),

$$
\left.\mu \mathcal{H o m}\left(\Phi_{1} \mathbb{Z}_{N}, \Phi_{1} \mathbb{Z}_{N}\right)\right|_{S^{*} X}=\left.\phi_{1 *} \mu \mathcal{H o m}\left(\mathbb{Z}_{N}, \mathbb{Z}_{N}\right)\right|_{S^{*} M}=\mathbb{Z}_{\mathbb{T}_{N^{\prime}}}
$$

we deduce that $\mathcal{L}$ has rank one and is concentrated in some (yet undetermined) cohomological degree. We compute (as always, above we write Hom for the derived functor; the final equality below would more precisely be written as a quasi-isomorphism between the complex on the left and a chain complex computing cohomology, though for the present purposes it is enough to know just the isomorphism at the level of cohomology groups):

$$
\begin{aligned}
\operatorname{Hom}_{N^{\prime}}\left(\mathbb{Z}_{N^{\prime}}, \mathcal{L}\right) & =\operatorname{Hom}_{M}\left(\mathbb{Z}_{M}, \Phi_{1} \mathbb{Z}_{N}\right) \\
& =\operatorname{Hom}_{M}\left(\left(\Phi_{1}\right)^{-1} \mathbb{Z}_{M}, \mathbb{Z}_{N}\right)
\end{aligned}
$$




$$
\begin{aligned}
& =\operatorname{Hom}_{M}\left(\mathbb{Z}_{M}, \mathbb{Z}_{N}\right) \\
& =H^{*}(N) .
\end{aligned}
$$

As $\mathcal{L}$ is in a single cohomological degree, the cohomological amplitude of $\operatorname{Hom}_{N^{\prime}}\left(\mathbb{Z}_{N^{\prime}}, \mathcal{L}\right)$ is at most $\operatorname{dim} N^{\prime}$. Thus, we conclude $\operatorname{dim} N^{\prime} \leqslant \operatorname{dim} N$; reversing the argument shows that this is in fact an equality. Now it follows, since the cohomological amplitude of $\operatorname{Hom}_{N^{\prime}}\left(\mathbb{Z}_{N^{\prime}}, \mathcal{L}\right)$ is exactly $\operatorname{dim} N^{\prime}$ and contains a nonzero term in degree zero, that $\mathcal{L}$ must be in degree zero. Being a rank one, degree zero local system with a section, $\mathcal{L}$ is trivial.

COROLLARY 12. There is an isomorphism of rings $H^{*}(N, \mathbb{Z}) \cong H^{*}\left(N^{\prime}, \mathbb{Z}\right)$.

Proof. $H^{*}(N, \mathbb{Z})=\operatorname{Hom}_{N}(\mathbb{Z}, \mathbb{Z})=\operatorname{Hom}_{M}\left(\mathbb{Z}_{N}, \mathbb{Z}_{N}\right)=\operatorname{Hom}_{M}\left(\Phi_{1} \mathbb{Z}_{N}\right.$, $\left.\Phi_{1} \mathbb{Z}_{N}\right)=\operatorname{Hom}_{M}\left(\mathbb{Z}_{N^{\prime}}, \mathbb{Z}_{N^{\prime}}\right)=\operatorname{Hom}_{N^{\prime}}(\mathbb{Z}, \mathbb{Z})=H^{*}\left(N^{\prime}, \mathbb{Z}\right)$.

REMARK 7. Note we used essentially that $\Phi_{1}$ carries $\operatorname{loc}(N) \rightarrow \operatorname{loc}\left(N^{\prime}\right)$, which in turn used essentially that $M$ was noncompact. Indeed, the above corollary is false otherwise: if $M$ is a projective space, then light shined from the origin $N$ recollects along the hyperplane at infinity $N^{\prime}$. Famously, this kind of counterexample is very special [Bot], perhaps suggesting that while the compact case is more complicated, it may not be much more complicated.

COROLlary 13. The map $\Phi_{1}: \operatorname{loc}(N) \rightarrow \operatorname{loc}\left(N^{\prime}\right)$ respects the standard $t$-structure.

Proof. For a local system $\mathcal{L} \in \operatorname{loc}(N)$, the sheaf $\mu \mathcal{H}$ om $\left(\mathbb{Z}_{N}, \mathcal{L}\right)$ is locally constant with the same stalks as $\mathcal{L}$. By Equation (4) and Lemma 11, we have

$$
\begin{aligned}
\left.\phi_{1 *} \mu \mathcal{H} \operatorname{om}\left(\mathbb{Z}_{N}, \mathcal{L}\right)\right|_{S^{*} M} & =\left.\mu \mathcal{H} \operatorname{Hom}\left(\Phi_{1} \mathbb{Z}_{N}, \Phi_{1} \mathcal{L}\right)\right|_{S^{*} M} \\
& =\left.\mu \mathcal{H} \operatorname{Hom}\left(\mathbb{Z}_{N^{\prime}}, \Phi_{1} \mathcal{L}\right)\right|_{S^{*} M} .
\end{aligned}
$$

But the left-hand side is the image under a diffeomorphism of a pullback of $\mathcal{L}$ and the right-hand side is a pullback of $\Phi_{1} \mathcal{L}$.

REMARK 8. Thus, $\Phi_{1}: s h_{\mathbb{T}_{N}}(M) \rightarrow s h_{\mathbb{T}_{N^{\prime}}}(M)$ respects any perverse $t$-structure. Compare [BEY].

COROLlaRY 14. $\Phi_{1}\left(\mathbb{Z}_{M \backslash N}\right) \cong \mathbb{Z}_{M \backslash N^{\prime}}$.

Proof. Apply $\Phi_{1}$ to the triangle $\mathbb{Z}_{M \backslash N} \rightarrow \mathbb{Z}_{M} \rightarrow \mathbb{Z}_{N} \stackrel{[1]}{\rightarrow}$. 
COROLlaRY 15. The following diagram commutes.

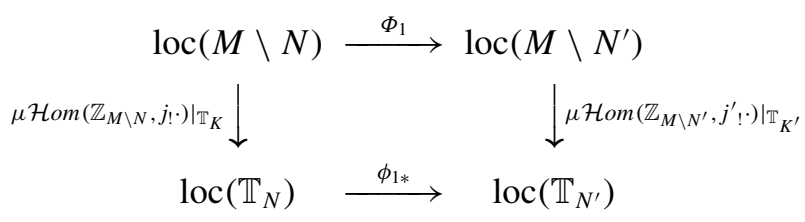

Proof. This follows from Equation (4), using Corollary 14 to trade $\Phi_{1} \mathbb{Z}_{M \backslash N}$ for $\mathbb{Z}_{M \backslash N^{\prime}}$

Let us conjugate $\phi_{1}$ by the geodesic flow. Note that geometrically this flow carries $\mathbb{T}_{N}$ and $\mathbb{T}_{N^{\prime}}$ to inward conormals to closed tubular neighborhoods $\operatorname{Nbd}(N)$ and $\operatorname{Nbd}\left(N^{\prime}\right)$. As we noted in Corollary 5, the sheaf quantization of these small isotopies, respectively, carry $\mathbb{Z}_{M \backslash N} \rightarrow \mathbb{Z}_{M \backslash N b d(N)}$ and $\mathbb{Z}_{M \backslash N^{\prime}} \rightarrow$ $\mathbb{Z}_{M \backslash N b d\left(N^{\prime}\right)}$.

Let us denote the conjugated isotopy by $\widetilde{\phi}_{1}$ and its sheaf quantization by $\widetilde{\Phi}_{1}$. Likewise, we denote the Legendrians formed by inward conormals to $N b d(N)$ and $\operatorname{Nbd}\left(N^{\prime}\right)$ as $\widetilde{\mathbb{T}}_{N}$ and $\widetilde{\mathbb{T}}_{N^{\prime}}$.

Conjugating everything in sight, we find $\widetilde{\Phi}_{1} \mathbb{Z}_{M \backslash N b d(N)} \cong \mathbb{Z}_{M \backslash N b d\left(N^{\prime}\right)}$ and the following commuting diagram:

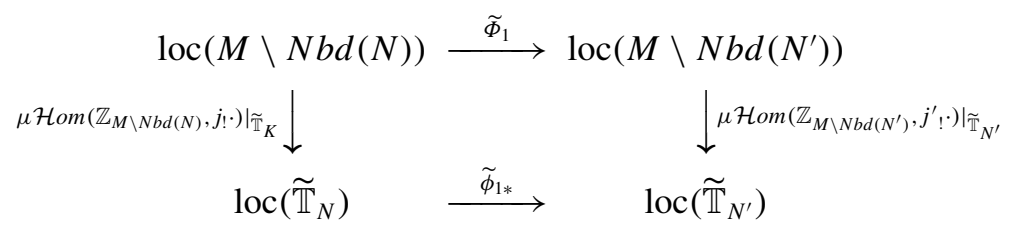

Now recall from Lemma 9 that $\mu \mathcal{H} \operatorname{om}\left(\mathbb{Z}_{M \backslash N b d(N)}, j ! \mathcal{L}\right)$ is supported on the $T_{M \backslash N b d(N)}^{+} M$, that is, the union of $M \backslash N b d(N)$ with the conormals which point into $\operatorname{Nbd}(N)$ and that, in addition, on this locus, it pulled back from $\mathcal{H o m}\left(\mathbb{Z}_{M \backslash N b d(N)}, j_{!} \mathcal{L}\right)$ under the projection to the zero section. A standard calculation shows that $\mathcal{H o m}\left(\mathbb{Z}_{M \backslash N b d(N)}, j_{!} \mathcal{L}\right)=j_{*} \mathcal{L}$. The conormals in $T_{M \backslash N b d(N)}^{+} M$ serve as a collar for $M \backslash N b d(N)$, so in all we have a canonical identification $\left.\left.\mu \mathcal{H} \operatorname{com}\left(\mathbb{Z}_{M \backslash N b d(N)}, j_{!} \mathcal{L}\right)\right|_{\widetilde{\mathbb{T}}_{K}} \cong j_{*} \mathcal{L}\right|_{N b d(N)}$.

As $\widetilde{\Phi}_{1}$ is the sheaf quantization of an isotopy which is constant over some locus in $M$ (recall $N$ is compact and $M$ is not), it preserves the stalks over this locus and, hence, carries local systems in the nonderived sense (that is, with cohomology sheaves only in degree zero) on $M \backslash N b d(\underset{\sim}{N})$ to the objects of the same kind on $M \backslash N b d\left(N^{\prime}\right)$. Now choose a point on $\widetilde{\mathbb{T}}_{N}$, a path $s \rightsquigarrow m$ in $M \backslash \operatorname{Nbd}(N)$ and a path $\phi_{1}(s) \rightsquigarrow m$ in $M \backslash N b d\left(N^{\prime}\right)$, where we have identified $\widetilde{T}_{N}$ with $\partial \operatorname{Nbd}(N)$ and, likewise, for $N^{\prime}$. These paths give commuting forgetful functors to $\mathbb{Z}$-modules; taking endomorphisms, we conclude the following: 
THEOREM 16. The isomorphism $\mathbb{Z}\left[\pi_{1}(M \backslash N b d(N))\right] \cong \mathbb{Z}\left[\pi_{1}\left(M \backslash N b d\left(N^{\prime}\right)\right)\right]$ extends to a commutative diagram

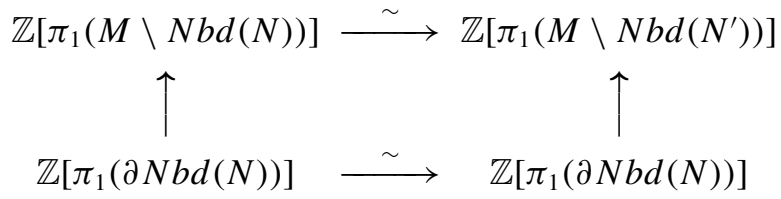

Here, the top horizontal arrow is induced by $\widetilde{\Phi}_{1}$, the bottom horizontal arrow is induced by $\widetilde{\phi}_{1}$, and the vertical arrows are induced by the topological inclusions.

REMARK 9. One can make this argument without first perturbing by the Reeb flow; however, in this case, the interpretation of the conormals as a collar would be less transparent, and, moreover, one would have to contemplate the microlocalization along a locus of codimension greater than one, for which the relevant Fourier transform is slightly harder to compute.

To finish the proof of Theorem 1, note that by hypothesis, $\phi$ carries meridians to meridians and longitudes to longitudes. Thus, we conclude by the above result and [Wal].

We turn to the proof of Theorem 2. What we need to show is that, even without assuming that the isotopy is parameterized, the longitude and meridian of $\mathbb{T}_{K}$ are carried up to signs to the longitude and meridian of $\mathbb{T}_{K}^{\prime}$. In fact, we will show this for a possibly multiple-component link, but we can only conclude the stated result regarding being equivalent or mirror in the case of knots, as the signs may be different for each component of the link.

Let $K$ be a component of $L$, and $K^{\prime}$ the corresponding component of $L^{\prime}$. Since $\Phi \mathbb{Z}_{K}=\mathbb{Z}_{K^{\prime}}$ and $\Phi$ carries $\operatorname{loc}(K)$ to $\operatorname{loc}\left(K^{\prime}\right)$, it must be that $\Phi$ carries nontrivial local systems on $K$ to nontrivial local systems on $K^{\prime}$. Let $\mathcal{L}$ and $\Phi \mathcal{L}=\mathcal{L}^{\prime}$ be such local systems. The meridian of $\mathbb{T}_{K}$ can be characterized, up to sign, as the primitive class in $\pi_{1}\left(\mathbb{T}_{K}\right)$ for which the holonomy of $\mu \mathcal{H o m}\left(\mathbb{Z}_{K}, \mathcal{L}\right)$ is trivial. Note $\phi_{*} \mu \mathcal{H}$ om $\left(\mathbb{Z}_{K}, \mathcal{L}\right)=\mu \mathcal{H} \operatorname{om}\left(\Phi \mathbb{Z}_{K}, \Phi \mathcal{L}\right)=\mu \mathcal{H}$ om $\left(\mathbb{Z}_{K^{\prime}}, \mathcal{L}^{\prime}\right)$. We conclude that $\phi$ carries the meridian of $K$ to the meridian of $K^{\prime}$ up to sign. Having found the meridians, the longitude of $K$ can be characterized up to sign as the primitive class in $\pi_{1}\left(\mathbb{T}_{K}\right)$ which goes to zero in the quotient of $H_{1}\left(\mathbb{R}^{3} \backslash L, \mathbb{Z}\right)$ by all meridians other than the meridian of $K$.

\section{Acknowledgements}

This project was inspired by discussions with Christopher Cornwell and Lenhard $\mathrm{Ng}$, in the wonderful environment of the Mittag-Leffler institute. 
I also thank Laura Starkston and Jacob Tsimerman for helpful conversations and Harold Williams for repeated explanations regarding the difference between finite and infinite rank local systems. I am partially supported by NSF DMS-1406871.

\section{References}

[Abo] M. Abouzaid, 'Nearby Lagrangians with vanishing Maslov class are homotopy equivalent', Invent. Math. 189(2) (2012), 251-313.

[AK] M. Abouzaid and T. Kragh, 'Simple homotopy equivalence of nearby Lagrangians', Acta Mathematica 220(2) (2018), 207-237.

[BBD] A. Beilinson, J. Bernstein and P. Deligne, 'Faisceaux pervers', Astérisque 100 (1982), 5-171.

[BEY] Y. Berest, A. Eshmatov and W.-K. Yeung, 'Perverse sheaves and knot contact homology', Comptes Rendus Mathematique 355(4) (2017), 378-399.

[BO] A. Bondal and D. Orlov, 'Reconstruction of a variety from the derived category and groups of autoequivalences', Compositio Math. 125(3) (2001), 327-344.

[Bot] R. Bott, 'On manifolds all of whose geodesics are closed', Ann. of Math. (2) 60 (1954), 375-382.

[Chi] S.-F. Chiu, 'Non-squeezing property of contact balls', Duke Mathematical Journal 166(4) (2017), 605-655.

[CELN] K. Cieliebak, T. Ekholm, J. Latschev and L. Ng, 'Knot contact homology, string topology, and the cord algebra', Journal de l'École polytechnique-Mathématiques 4 (2017), 661-780.

[Cor1] C. R. Cornwell, 'KCH representations, augmentations, and A-polynomials', Journal of Symplectic Geometry 15(4) (2017), 983-1017.

[Cor2] C. R. Cornwell, 'Knot contact homology and representations of knot groups', J. Topol. 7(4) (2014), 1221-1242.

[ENS] T. Ekholm, L. Ng and V. Shende, 'A complete knot invariant from contact homology', Inventiones mathematicae 211(3) (2018), 1149-1200.

[FSS] K. Fukaya, P. Seidel and I. Smith, 'Exact Lagrangian submanifolds in simply-connected cotangent bundles', Invent. Math. 172(1) (2008), 1-27.

[Gr] J. Gray, 'Some global properties of Contact structures', Ann. of Math. (2) 69(2) (1959), $421-450$.

[GL] C. Gordon and J. Luecke, 'Knots are determined by their complements', J. Amer. Math. Soc. 2(2) (1989), 371-415.

[GLi] C. Gordon and T. Lidman, 'Knot contact homology detects cabled, composite, and torus knots', Proceedings of the American Mathematical Society 145(12) (2017), 5405-5412.

[Gui] S. Guillermou, 'Quantization of conic Lagrangian submanifolds of cotangent bundles'. Preprint, 2012, arXiv:1212.5818.

[Gui2] S. Guillermou, 'The Gromov-Eliashberg theorem by microlocal sheaf theory'. Preprint, 2013, arXiv:1311.0187.

[Gui3] S. Guillermou, 'The three cusps conjecture'. Preprint, 2016, arXiv:1603.07876.

[GKS] S. Guillermou, M. Kashiwara and P. Schapira, 'Sheaf quantization of Hamiltonian isotopies and applications to nondisplaceability problems', Duke Math. J. 161(2) (2012), 201-245.

[Her] M. Hertwick, 'A counterexample to the isomorphism problem for integral group rings', Ann. of Math. (2) 154(1) (2001), 115-138. 
[Hig] G. Higman, 'The units of group-rings', Proc. Lond. Math. Soc. (2) 46 (1940), 231-248.

[HS] J. Howie and H. Short, 'The band-sum problem', J. Lond. Math. Soc. (2) 31(3) (1985), 571-576.

[KS] M. Kashiwara and P. Schapira, Sheaves on Manifolds, Grundlehren Math. Wiss., 292 (Springer, 1990).

[N1] D. Nadler, 'Microlocal branes are constructible sheaves', Selecta Math. (N.S.) 15(4) (2009), 563-619.

[Ng3] L. Ng, 'Framed knot contact homology', Duke Math. J. 141(2) (2008), 365-406.

[Orl] D. Orlov, 'Derived categories of coherent sheaves and equivalences between them', Russian Math. Surveys 58(3) (2003), 511-591.

[STZ] V. Shende, D. Treumann and E. Zaslow, 'Legendrian knots and constructible sheaves', Inventiones mathematicae 207(3) (2017), 1031-1133.

[STWZ] V. Shende, D. Treumann, H. Williams and E. Zaslow, 'Cluster varieties from Legendrian knots'. Duke Mathematical Journal, Preprint, 2015, arXiv:1512.08942, to appear.

[STW] V. Shende, D. Treumann and H. Williams, 'On the combinatorics of exact Lagrangian surfaces'. Preprint, 2016, arXiv:1603.07449.

[Tam] D. Tamarkin, 'Microlocal condition for non-displaceablility', in Algebraic and Analytic Microlocal Analysis (Springer, 2013), 99-223.

[Wal] F. Waldhausen, 'On irreducible 3-manifolds which are sufficiently large', Ann. of Math. (2) 87(1) (1968), 56-88. 